\title{
Default-mode network activity and its role in comprehension and management of psychophysiological insomnia: A new perspective
}

\author{
Daniel Ruivo Marques ${ }^{\mathrm{a}, \mathrm{b},{ }^{*}}$, Ana Allen Gomes ${ }^{\mathrm{a}, \mathrm{b}}$, Vanda Clemente ${ }^{\mathrm{c}}$, \\ José Moutinho dos Santos ${ }^{\mathrm{c}}$, Miguel Castelo-Branco ${ }^{\mathrm{b}}$ \\ a Department of Education, University of Aveiro, Campus Universitário de Santiago, 3810-193 Aveiro, Portugal \\ b IBILI (FCT), Faculty of Medicine, University of Coimbra, Azinhaga de Santa Comba, Celas, 3000-548 Coimbra, Portugal \\ ' Sleep Medicine Centre, CHUC (Centro Hospitalar e Universitário de Coimbra), Quinta dos Vales, São Martinho do Bispo, $3046-853$ \\ Coimbra, Portugal
}

\section{Keywords:}

Insomnia

Hyperarousal

Default-mode network

Cognitive-behavioral therapy for insomnia

\begin{abstract}
A B S T R A C T
Psychophysiological insomnia (PI) is a common sleep disorder in which numerous variables interact. The mechanisms responsible for the etiology and maintenance of PI, though far from completely understood, point to the existence of hyperarousal of several systems. The frequent occurrence of ruminations and worries with a self-referential component (related or not with sleep complaints) during the pre-sleep period, and daytime wakefulness, seems to relate to the functions which have been associated with default-mode network (DMN) activity. This neural network seems to be involved in introspective thinking as well as emotional and episodic memory processing, among others. In this paper, we propose that PI may be conceptualized as a disorder associated with overactivity of some brain areas of DMN. Accordingly, it is also suggested that cognitive-behavioral therapy for insomnia (CBT-I), a kind of non-pharmacological treatment, may alter the function of this network, improving symptoms of patients, and overall quality of life.
\end{abstract} (C) 2014 Elsevier Ltd. All rights reserved.

\section{Introduction}

Approximately $10-15 \%$ of the general population has insomnia and this is considered as being one of the most common sleep disorders with an estimated incidence of 3-5\% of new cases each year (Drake \& Roth, 2006). Psychophysiological insomnia (PI) is a sleep disorder with clear-cut classification criteria in terms of medical

\footnotetext{
* Corresponding author. Departamento de Educação da Universidade de Aveiro, Campus Universitário de Santiago, 3810-193 Aveiro, Portugal. Tel.: +351234372428.

E-mail addresses: drmarques@ua.pt (D.R. Marques), ana.allen@ua.pt (A.A. Gomes), vandaclem@gmail.com (V. Clemente), josemoutinho@ netcabo.pt (J. Moutinho dos Santos), mcbranco@fmed.uc.pt (M. CasteloBranco).
}

diagnosis and covers complaints in starting, maintaining sleep, or experiencing non-restorative sleep. According to the second edition of International Classification of Sleep Disorders - ICSD-2 of the American Academy of Sleep Medicine (AASM, 2005), this is a diagnosis that includes high cognitive, physiological, and emotional arousal levels associated with negative conditioning between some stimuli or spatial/temporal cues and sleep behaviors.

As suggested in the literature, insomnia (particularly conditioned insomnia) seems to be a fluctuating disorder. This is evident when we analyze the amount of pathophysiologic models that have been proposed to explain it (Bootzin, 1972; Espie, Broomfield, MacMahon, Macphee, \& Taylor, 2006; Harvey, 2002; Kales, Caldwell, Preston, Healey, \& Kales, 1976; Lundh \& Broman, 2000; Morin, 1993; Ong, Ulmer, \& Manber, 2012; Perlis, Giles, 
Mendelson, Bootzin, \& Wyatt, 1997; Spielman, Caruso, \& Glovinsky, 1987). All these models, mainly psychological in their origins, gave consistency to the two hypotheses explaining insomnia development and maintenance processes: hyperarousal and failure to inhibit wakefulness, respectively (Perlis, Shaw, Cano, \& Espie, 2011). The hyperarousal hypothesis states that in patients with insomnia there is a widespread activation of several systems (e.g., cognitive, physiological, emotional, cortical), which consequently prevents the person to relax. The concept of hyperarousal was recognized to be of major importance in the understanding of PI. However, sleep researchers have not yet reached a consensus on which dimensions this concept covers (Riemann et al., 2010).

On the other hand, the failure to inhibit wakefulness account suggests that the difficulty in inhibiting activation typical of wakefulness period is the principal disturbing process in PI (Espie et al., 2006). In practice, it is feasible to accept the complementarity of both hypotheses (Perlis et al., 2011). It is likely that these two processes may relate to two distinct profiles of patients with insomnia. Although interesting, we do not have yet evidence to support this claim.

Many studies have concluded that bedtime is the period of day in which individuals are more available to deal with emotionally arousing cognitions (Harvey, 2005). For example, people tend to focus on the concerns regarding the organization and management of the following day, to elaborate a retrospective of the past day, to remember past traumatic events, or to anxiously anticipate the future, or generate negative expectations related to own sleep behavior, among many others (Watts, Coyle, \& East, 1994). Accordingly, it is easy to understand that intrusive and dysfunctional thoughts related with the self play a key role in PI.

As already mentioned, there are many models proposed for understanding the etiology and maintenance of PI. Nevertheless, it should be noted that the behavioral or psychological models are the most studied and well-known ones, with the added advantage, but also the challenge, of integrating neurobiological, biochemical, physiological, and even immunological variables (Talbot \& Harvey, 2010).

In this article, we will present some provisional ideas suggesting that the new developments in neuroscience, in particular, pertaining to the brain's default-mode network (DMN) study will bring important advances for insomnia's conceptualization and treatment.

\section{The default-mode network}

The function of the DMN is currently one of the most studied topics in the field of cognitive neuroscience. In general, it concerns to a relatively well-defined set of brain areas which have a higher level of activation when the subjects are not focused on a specific external task mobilizing their explicit attentional resources (i.e., goal-oriented task or attention demanding task). For this reason, it is also called a task-negative network (Raichle \& Snyder, 2007). In spite of this, it is relevant to stress that DMN brain regions are also generally activated in attention demanding tasks albeit in a lesser extent (the exception is when the individuals perform tasks requiring self-referential processing).

The DMN has captured the interest of many scholars since during this "state of rest" the brain consumes identical energy resources compared with solving arithmetic problems tasks, for example (Snyder \& Raichle, 2012).

Several studies using the resting-state paradigm and focused particularly in DMN have shown that there are basic psychological functions associated with this neural network. The DMN is involved in behaviors such as mindwandering, recovery of past memories, planning/projection of future events, and consideration of perspective/ point of view of other individuals (theory of mind). All of these functions activate multiple regions within the DMN (Buckner, Andrews-Hanna, \& Schacter, 2008). Overall, they represent what the researchers refer to as the "internal modes of cognition". The resting-state experiments (i.e., in which the participant is asked to simply relax with eyes closed or open, as appropriate) allow to obtain an overview of the most active brain regions that a growing number of studies have shown to be related with each other (i.e., work interdependently), when the subject is focused only on their own psychological processes (Raichle \& Snyder, 2007). However, we must note that DMN constitutes just one of the many resting networks observed in the brain.

Although there is no full understanding about the functions inherent to DMN, several studies have converged in identifying the underlying brain structures. These regions include medial areas of the brain, comprising the ventral medial prefrontal cortex (MPFC), dorsal MPFC, medial temporal lobule, inferior parietal lobule (IPL), precuneus, posterior cingulate cortex (CPP)/retrosplenial cortex, and the hippocampal formation (Buckner et al., 2008). Nonetheless, it is worth noting that the current consensus is on MPFC, including anterior cingulate cortex (AAC), PCC, and IPL (Whitfield-Gabrieli \& Ford, 2012).

There are some studies suggesting that the DMN, instead of representing a cohesive and coherent organization, can be divided into specific sub-organizations. In an attempt to dissociate subsystems within DMN, AndrewsHanna, Reidler, Sepulcre, Poulin, and Buckner (2010) observed that this network can be subdivided into two main components, taking into account the self-reference and temporal orientation variables. These authors reported that one component is the medial prefrontal cortex system - including the dorsal prefrontal medial dorsal, the temporo-parietal junction, the lateral temporal cortex, and the temporal pole; the other component is the temporal lobe subsystem which includes the medial ventral prefrontal cortex, posterior inferior parietal lobe, retrosplenial cortex, parahippocampal cortex and the hippocampal formation, and is mobilized when individuals engage in decisions that require mental simulations based on memory. In future-oriented cognitions the two subsystems are simultaneously mobilized, presumably to facilitate the construction of mental models that enable people to adapt to the environment.

Since the last decade, many scholars have sought to understand the patterns of response of the DMN in various neuropsychiatric disorders such as Alzheimer's disease, autism spectrum disorders, schizophrenia, attention deficit 
hyperactivity disorder, depressive disorders, anxiety disorders, among others (Broyd et al., 2009; WhitfieldGabrieli \& Ford, 2012).

In the case of sleep disorders and PI, in particular, the study of the functional impact of DMN activity seems to be of utmost importance. Resting-state studies appear to be a very reliable and valid biological paradigm to examine the "rest activity" of these patients because it simulates what appears to happen every evening (e.g., in a typical night) prior to sleep-onset, for instance.

\section{Insomnia as a self-referential processing disorder: the DMN dysfunction pathway}

Until now, we enlightened that DMN has an important role in many human functions and may be disrupted in several disorders. The most studied disorders have been the Alzheimer's disease, major depression, anxiety disorders, schizophrenia, autism, and attention-deficit/ hyperactivity disorder. However, the DMN dysfunction hypothesis has been examined also in other disorders beyond neuropsychiatric ones (e.g., Kornelsen et al., 2013; Liu et al., 2013; Tregellas et al., 2011; Violante et al., 2012; Wolf et al., 2012). For this article purpose, we will outline major depression and anxiety disorders findings since depressive and anxiety symptoms are common in PI patients (AASM, 2005).

In major depression it has been observed a general pattern of activation in DMN areas (Greicius et al., 2007; Marchetti, Koster, Sonuga-Barke, \& Raedt, 2012), even when the patients are performing an attention demanding task (Sarsam, Parkes, Roberts, Reid, \& Kinderman, 2013). This may explain the dysfunctional cognitive rumination reported by these individuals (Anticevic et al., 2013). In patients with varied anxiety disorders it has been found a failure in normal DMN deactivation, and maintenance of intense DMN activity when these individuals are exposed to neutral stimuli (Zhao et al., 2007). In posttraumatic stress disorder, it was observed an impairment in DMN according to a connectivity functional analysis (Daniels et al., 2010). In a study encompassing a group of generalized anxiety disorder patients, a frequent comorbid disorder in PI (Bélanger, Morin, Langlois, \& Ladouceur, 2004), it was found that either patients or healthy controls activated the MPFC and AAC - pivotal areas of DMN - when they were exposed to anxiogenic stimuli; however, the clinical group kept the activation in these brain regions when in restingstate (Paulesu et al., 2009). All the studies cited were performed with functional magnetic resonance imaging (fMRI).

Notwithstanding, we must stress that the results of the studies mentioned so far are largely dependent of sample sizes, experimental designs, data analysis techniques, among many other factors.

In healthy individuals it seems that DMN plays a key role in mood regulation as well. A study by Killingsworth and Gilbert (2010) reinforced the idea that there is an emotional cost associated with DMN activity (regardless of its benefits and evolutionary/adaptive advantages). This cost refers to the negative correlation that seems to exist between levels of self-reported happiness and activity in this neural network. In summary, the more the activity in the DMN (i.e., self-referential processing), the greater the likelihood of individuals reporting psychological distress.

Before we put forward some hypotheses based on our proposed "DMN dysfunction pathway in insomnia", we will refer some studies that recently have emerged on DMN, sleep and insomnia relationships.

Larson-Prior et al. (2006) studied the association between cerebral electrical activity related to introspective and relaxing states (i.e., alpha waves) and BOLD (blood oxygenation level dependent) signal during two restingstate conditions (eyes closed during 5 and 20 min periods) in a sample of 34 healthy individuals. The results showed a significant association between these two signal types, particularly located in PCC. In another study with 10 healthy participants, the same authors studied the functional connectivity between DMN in wakefulness state and NREM sleep stages using fMRI and simultaneous EEG (electroencephalography). The main conclusion was that functional connectivity patterns within DMN from wakefulness to sleep is maintained (Larson-Prior et al., 2009). On the other hand, a study by Horovitz et al. (2009) with healthy individuals found that during normal sleep there are significant modifications in DMN activity, in particular, a reduced correlation between anterior and posterior components. It may happen that this pattern does not occur in PI patients.

Gujar, Yoo, Hu, and Walker (2010) recognized that sleep deprivation in non-clinical populations altered activity in the DMN. Sämann et al. (2011) recruited a sample of 25 healthy volunteers and investigated brain's default mode network from wakefulness to slow wave sleep. They found that as sleep depth increases, some DMN's brain areas such as PCC, retrosplenial cortex, parahippocampal gyrus, and MPFC, decreased their activity. Other research by De Havas, Parimal, Soon, and Chee (2012) evaluated functional connectivity among DMN regions before and after $24 \mathrm{~h}$ sleep deprivation $(n=26)$. After sleep deprivation, there was a reduction in functional connectivity in DMN. Rao et al. (2012, October) verified that it only takes one sleepless night to disrupt the way in which hippocampus and DMN regions communicate. In the meantime, when the subjects recovered the "lost sleep", the pattern becomes normal again.

The only published studies we found specifically about DMN and its relationship to insomnia (at least to our knowledge) were the ones by Hasler et al. (2013) and Drummond et al. (2013).

Hasler et al. (2013) investigated the functional connectivity between areas of the DMN in a sample of patients with PI $(n=53)$ and in a sample of good sleepers $(n=52)$ in four different times of the day: in the morning after waking-up; in wakefulness at the end of the day/early evening; during NREM sleep; and during NREM sleep after sleep restriction. It was verified that both groups were not distinct regarding functional connectivity in the morning period after waking-up. However, in the other three moments of the day the groups showed differences. The control group evidenced higher correlation values between PCC and MPFC during wakefulness at evening and NREM sleep. The clinical group showed higher magnitude 
correlations in these brain regions after sleep restriction, comparatively to the control group. Finally, insomnia patients revealed higher magnitude associations between PCC and inferior parietal cortex, except for post-sleep restriction NREM sleep. This research was performed through [18F]fluorodeoxyglucose positron emission tomography.

The other study on insomnia (albeit not focused explicitly in DMN) aimed to compare the performance on a test of working memory (n-back working memory test) in a group of patients with PI $(n=25)$, and a group of matched individuals from the general population $(n=25)$. Although there have been no differences between the groups in behavioral performance, it was found that patients with PI did not recruit successfully the same brain regions involved in the memory task compared to the control group. Interestingly, it was also found that insomnia patients were not so able to deactivate brain regions related to the DMN when compared to the control group. This effect was more pronounced when the difficulty of the memory task increased (Drummond et al., 2013). This research was performed through fMRI.

Finally, it should be mentioned a study using simultaneous fMRI and EEG by Chen, Chang, Glover, and Gotlib (2014) concerned with resting-states study on insomnia. They analyzed the functional connectivity of 17 PI patients and 17 healthy control participants during rest and during the time the participants tried to fall asleep. It was found that there was an aberrant activation of the insula in PI patients. Besides, this insula activity was related to gamma activity in EEG and negative affect.

Noteworthy, we must underscore that the inclusion of actual sleep stages into the DMN model is problematic. It has been shown that there is a general metabolic decrease from waking to sleeping (not restricted to DMN areas); thus, brain activity and connectivity patterns differ fundamentally between waking and sleeping.

\subsection{Integrating DMN in current approaches to insomnia}

According to the still scarce literature on the role of the DMN in insomnia and sleep, we can draw some provisional explanatory links which shall be validated by future studies.

When the individual is in bed preparing for sleep it seems plausible that by the absence of tasks/external stimuli that require focused attention, the person focuses on its internal functioning by "default". According to the most well-known explanatory processes in insomnia (see Introduction), we can delineate two types of possible trajectories: (1) there is a significantly high level of arousal in DMN brain areas even during the day, and which tends to persist at night, and possibly during sleep stages - hyperarousal hypothesis (Riemann et al., 2010); (2) there is pronounced activation at bedtime particularly; with the approaching of night, and in the absence of explicit tasks to mobilize the attention of the subject, the attentional focus will be on self-referential processes (Harvey, 2005) which involve the activation of the DMN. That is, the other neural networks will reduce their activity, whereas DMN will not be so deactivated. The activity in DMN might inhibit brain structures underlying sleep induction - failure to inhibit wakefulness hypothesis (Espie et al., 2006). According to these considerations, ruminations and concerns will occupy attentional resources of the individuals, thereby disrupting their sleep. The way the person copes with these pathophysiological processes will depend on several variables commonly referred in the literature such as sleep anxiety, sleep effort, cognitive and behavioral coping strategies, among others (Espie et al., 2006; Harvey, 2002, 2005; Morin, 1993; Perlis et al., 2011).

In insomnia disorder it is conceivable that one can interpret the dysfunction of DMN in two complementary ways (which may reflect the different cluster categories of patients with PI): (1) lack of suppression of irrelevant processes when performing demanding tasks (as also observed in schizophrenia, for instance), and (2) lack of suppression primarily assigned to abnormally intense cognitive activity (as suggested in the context of depression) (Anticevic et al., 2013; Whitfield-Gabrieli \& Ford, 2012). We hope that future research can shed light on these hypotheses.

\subsection{The "head button" that never switches off}

In clinical practice it is common to hear insomnia patients saying that they should have a button/switch in their heads that they could shut down to fall asleep quickly (i.e., avoiding the flood of thoughts related to the self, and generally with a negative emotional valence). Perhaps research regarding the DMN may shed light on understanding of these cognitive activation complaints in two ways: First, this "button" would not necessarily be categorical (i.e., only with two options: on - wakefulness and off - sleep), but instead it would be something more like a dimensional continuum; on the other hand, could be a set of "buttons" as represented by various brain structures constituting DMN. These hypotheses seem to fit in the new theoretical developments of insomnia disorder (see, for example, the neurobiological model by Buysse, Germain, Hall, Monk, \& Nofzinger, 2011).

\subsection{One step ahead in insomnia}

We have discussed the putative interplay between insomnia disorder and DMN hitherto. However, a caveat should be underlined. We would like to stress that the perspective we propose (i.e., DMN dysfunction in insomnia) should be seen as a useful theoretical framework (though complementary) to the existing models and theories. In this vein, the hypothesized DMN dysfunction in insomnia resembles the insomnia's REM sleep instability account for insomnia proposed by Riemann et al. (2012). This hypothesis establishes that insomnia patients are prone to more nocturnal micro- and macro-awakenings because REM sleep is the most aroused state that individual experience when they are sleeping. This account reinforces the hyperarousal concept in insomnia. One must note that the authors stated carefully that this is not a new theory but rather a new hypothesis that may contribute to a better understanding of insomnia alongside with the current models. 
Given the foregoing, it can be hypothesized that persistent arousal in PI may be due to in part a hyperactivation in brain areas related to DMN, before or during sleep, and even during the period of wakefulness (i.e., during the day) (Nofzinger, 2010). We assume that this hyperarousal mechanism relates with behavioral characteristics that cover many domains (i.e., cognitive, physiological, emotional) which are interdependent. Although we are interested in measuring its neurobiological correlates, we argue that the cognitive arousal experienced by insomnia patients is inextricably linked to the biological underpinnings (Perlis et al., 1997), and the respective need to be studied as directly as possible, because of the mental or psychological functions which have been attributed to the DMN. This would allow, as is done for other disorders, to more closely test if activity patterns within the DMN are responsive to different kinds of treatment, particularly cognitive-behavioral therapy for insomnia (CBT-I) which is a well-established clinical intervention for insomnia (Morgenthaler et al., 2006; Whitfield-Gabrieli \& Ford, 2012). Currently, and taking into account the putative functions of the DMN, we are interested in objectively testing into which extent autobiographical activating stimuli generated by the subjects and related to different temporal orientations (i.e., past/present and future) drive different areas of the DMN. In insomnia research, this issue seems to be relevant since there are significant interindividual differences, and some patients report many activating cognitions related to what they have to do next day (future), while others patients refer more aspects related with situations about past and/or present events (ruminations). In addition, in accordance with what we mentioned earlier, we will explore the impact of eye opening and closure on patterns of neuronal activation in areas related to the DMN, given that this affects the ongoing alpha rhythm (reflecting visual idleness) state level and the link between visual processing and the DMN (Barry, Clarke, Johnstone, Magee, \& Rushby, 2007; Knyazev, SlobodskojPlusnin, Bocharov, \& Pylkova, 2011; Xu et al., 2014). Consequently, these results should be compared with a control group without sleep problems. It is also possible that there may be an association between cortical areas that mediate the different types of temporal orientation, the fact that the participants have their eyes closed or not, and the related physiological state. In the context of the clinical phenomenology of insomnia this seems very relevant. We think that there may be important differences in temporal patterns of activity related to introspection over time in patients with PI compared to other neuropsychiatric disorders and control samples. It was already mentioned that the DMN hyperactivation observed in major depression disorder seems to relate to ruminative activity. Given that depressive disorders are one of the most common comorbid conditions associated with PI, we wonder if the PI pattern will be the same or if, on the other hand, the component relating to the processing of "future" concerns will be more active, giving consistency to what seems to be more apparent in clinical practice (cf. Integrating DMN in current approaches to insomnia) (Buckner et al., 2008; Whitfield-Gabrieli \& Ford, 2012). Additionally, a relevant topic to explore in the future is to analyze whether DMN abnormalities observed in insomnia might also explain DMN abnormalities verified in patients with psychiatric disorders.

\section{Specificities regarding the study of the DMN in insomnia}

Taking into account some main characteristics of PI (e.g. intrusive thoughts/cognitive arousal) and the functions that appear to be hypothetically related to the DMN, we think it is very important in clinical practice and neurobiological research to study how the regions of DMN modulate their activity in these patients compared with matched control samples taken from general population, without sleep complaints or other neuropsychiatric disorders. It should be outlined that the scientific literature indicates that patients with insomnia are ten times more likely to report their pattern of cognitive arousal as more disturbing than the physiological one (Lichstein \& Rosenthal, 1980). Moreover, studies of functional connectivity (i.e., assessment of direction and magnitude of relationships among different brain areas) allow us to understand how the different brain structures communicate with each other. Further, the use of active task paradigms enabling the study of neural activation according to different blocks of stimuli, for example, is also an important avenue for research. To achieve this, it is essential the use of neuroimaging techniques such as fMRI complemented with other traditional measures used in sleep medicine, including self-response questionnaires, actigraphy, and polysomnography (Whitfield-Gabrieli \& Ford, 2012).

Although it is important to evaluate the DMN in insomnia patients prior to bedtime or even during all sleep stages, we emphasize that the same studies must be performed during daytime, since PI seems to be a 24h disorder (Riemann et al. 2010). As Lundh (2005) states: "there is evidence that people's cognitive-emotional processes at restful moments during the day are representative of their presleep processes. This finding makes it possible to study the relevant cognitive-emotional processes by having the individual lie down on a bed for short periods in the laboratory during the day" (p.31).

In fMRI neuroimaging studies participants have to lie down for data acquisition. Contrary to the usual fMRI scannings, we posit that in insomnia patients the measurement time in the scanner should be extended (e.g., $60 \mathrm{~min}$ ). This is important because the prolonged exam may favors boredom - and insomnia patients may or may not fall asleep. However, it is important to underline that this extended duration of scans may bring confounds to the data as the brain states vary over time even in healthy control individuals (Tagliazucchi \& Laufs, 2014). Whenever possible, one should control this issue through collecting EEG data simultaneously or using simple tasks such as psychomotor vigilance tests (Sämann et al., 2010). On the other hand the scanning environment might lead to higher levels of arousal. In this vein, it may be considered as a "stimulus provocation paradigm" variant (Linden, 2006). Other cluster of insomnia patients where the maladaptive conditioning principles between their habitual bed/bedroom and sleep behavior are most evident may actually sleep in the scanner. It is therefore 
no surprise that some of these patients may at least doze in fMRI experiments since it is a new environment. One must note that this paradigm is well suited for research questions that are not focused in studying the performance of the individuals in attention-demanding tasks. In the latter case, the scanning times must be curtailed.

In insomnia, it seems to make sense to study the DMN either with eyes open or eyes closed during the restingstate, since even before falling asleep, when individuals are in bed, there is an inter-individual variability in the tendency for attempting to sleep (i.e., sleep effort) and, as such, keeping eyes closed. On the other hand, given the cognitive load hypothetically mediated by DMN, there might be a tendency to keep eyes open as a reflex response to daydreaming/mindwandering, even if the external environment remains dark or without any light source.

Given the particularities of insomnia, several experimental design strategies are viable. Insomnia groups should be compared against control groups (i.e., groups without sleep complaints or disorders), and if possible paired casecontrol studies (whenever possible). The aim will be to compare each patient sample against her/his paired control participant. According to the extent literature and our own clinical experience it seems plausible that in PI there is a considerable variability in many important processes among patients (e.g., cognitive processes as selective attention, dysfunctional beliefs, coping strategies, among many others).

In short, as major advantages and limitations of DMN research in insomnia we highlight:

Advantages:

(i) It may account to explain at least partially sleep-onset complaints, intermediate awakenings, and daytime (dys)function;

(ii) It suits for the research during daytime, nighttime and sleep periods;

(iii) It might help to clarify and discriminate the role of the two main processes in PI (i.e., hyperarousal and failure to inhibit wakefulness);

(iv) It will help in the validation of evidence-based psychotherapies according to a neurobiological approach, resembling what is being studied in other disorders (e.g., anxiety and depressive disorders) (cf. Implications for treatment section);

(v) Strengthens the link between methodological approaches in cognitive neuroscience and psychological science knowledge.

\section{Limitations:}

(i) It cannot be a model or theory that explains insomnia disorder per se;

(ii) It is a new research topic. Thus, there is scarce evidence (few studies yet) of the DMN dysfunction in insomnia;

(iii) As the most used tool to study DMN is fMRI, the development in this field might be delayed because of cumbersome costs and resistance of some patients in voluntarily collaborating in a lengthy study.

\section{Implications for treatment}

If future research validates the hypotheses that (a) there is a significant correlation between activity of DMN's regions and the cognitive experiences that patients often report, and (b) that this association is significantly different from control samples (i.e., individuals without sleep disorders), psychotherapeutic techniques derived from cognitive therapies may be strategies to favor in the management of insomnia, giving strength to the idea that all insomnia treatment packages must contain explicitly cognitive techniques. Current recommendations regarding insomnia's treatment suggest that cognitive strategies are effective only when they are included in a multimodal approach (i.e., along with behavioral techniques such as stimulus control therapy, sleep restriction therapy, and relaxation training (Morgenthaler et al., 2006)). Therefore, there is no evidence to recommend cognitive therapy as the only methodology for insomnia's treatment. However, recent studies have begun to pinpoint to the effectiveness of this therapy by itself (Morin, Harvey, \& Bélanger, 2011). Moreover, this research may help to validate strategies based on mindfulness and acceptance approaches, which will surely contribute to the debate about the integration of third generation CBT with traditional CBT-I.

Still, if CBT-I induces significant objective changes in activity of these brain areas involved in the DMN, the relevance of so-called empirically validated psychotherapies will gain a new role (Linden, 2006). We believe this will help to validate, from another perspective (i.e., neurobiological), the psychological interventions that have been proven effective in treatment of many neuropsychiatric disorders, including insomnia (Bastien, 2011).

\section{Concluding remarks}

We think that the model of impaired DMN function and the raised provisional hypotheses we propose in this paper, can be integrated in other comprehensive models of insomnia that already exist (e.g., Espie's psychobiological inhibition model). In clinical practice, it is common to observe that different explanatory models can be used successfully to different patients with PI diagnosis.

It will be helpful to foster future research on the putative role of different functional clusters within DMN in PI patients with respect to the temporal orientation of cognitive processes (i.e., past, present, and future) since there is evidence suggesting that these are functionally segregated within the DMN (Andrews-Hanna et al., 2010; Schacter, Addis, \& Buckner, 2007).

We posit that the study of DMN in the field of sleep medicine (particularly in insomnia) will bring new translational research challenges to the field of sleep neuroscience, in particular, concerning to the conceptual link with clinical practice (Wamsley \& Stickgold, 2011).

Ultimately, perhaps the results obtained with the resting-state paradigm may help improve the understanding of PI etiological models and reconcile key concepts that seem to underlie its clinical expression: hyperarousal and failure to inhibit wakefulness processes (Perlis et al., 2011). 
Snyder and Raichle (2012) refer that resting-state neuroimaging studies have been motivated essentially by neurobiological questions and much less by clinically oriented cognitive science. It is our expectation that research regarding DMN in PI can bridge this gap.

\section{Acknowledgment}

First author has a PhD Grant from the Portuguese Foundation for Science and Technology (FCT - Fundação para a Ciência e a Tecnologia) with the following reference: SFRH/BD/77557/2011.

\section{References}

American Academy of Sleep Medicine. (2005). International classification of sleep disorders: Diagnostic and coding manual (2nd ed.). Westchester, IL: American Academy of Sleep Medicine.

Andrews-Hanna, J., Reidler, J., Sepulcre, J., Poulin, R., \& Buckner, R. (2010). Functional-anatomic fractionation of the brain's default network. Neuron, 65, 550-562. http://dx.doi.org/10.1016/j.neuron.2010.02.005.

Anticevic, A., Cole, M., Murray, J., Corlett, P., Wang, X.-J., \& Krystal, J (2013). The role of default network deactivation in cognition and disease. Trends in Cognitive Sciences, 16(12), 584-592. http:// dx.doi.org/10.1016/j.tics.2012.10.008.

Barry, R., Clarke, A., Johnstone, S., Magee, C., \& Rushby, J. (2007). EEG differences between eyes-closed and eyes-open resting conditions. Clinical Neurophysiology, 118, 2765-2773. http://dx.doi.org/10.1016/ j.clinph.2007.07.028.

Bastien, C. (2011). Insomnia: neurophysiological and neuropsychological approaches. Neuropsychological Review, 21, 22-40. http://dx.doi.org/ 10.1007/s11065-011-9160-3.

Bélanger, L., Morin, C., Langlois, F., \& Ladouceur, R. (2004). Insomnia and generalized anxiety disorder: effects of cognitive behavior therapy for gad on insomnia symptoms. Journal of Anxiety Disorders, 18(4), 561-571. http://dx.doi.org/10.1016/S0887-6185(03)00031-8.

Bootzin, R. (1972). Stimulus control treatment for insomnia. Proceedings of the American Psychological Association, 7, 395-396.

Broyd, S., Demanuele, C., Debener, S., Helps, S., James, C., \& SonugaBarke, E. (2009). Default-mode brain dysfunction in mental disorders: a systematic review. Neuroscience and Biobehavioral Reviews, 33 , 279-296. http://dx.doi.org/10.1016/j.neubiorev.2008.09.002.

Buckner, R., Andrews-Hanna, J., \& Schacter, D. (2008). The brain's default network: anatomy, function, and relevance to disease. Annals of New York Academy of Sciences, 1124, 1-38. http://dx.doi.org/10.1196/ annals.1440.011.

Buysse, D., Germain, A., Hall, M., Monk, T., \& Nofzinger, A. (2011). A neurobiological model of insomnia. Drug Discovery Today: Disease Models, 8(4), 129-137. http://dx.doi.org/10.1016/j.ddmod.2011.07.002.

Chen, M., Chang, C., Glover, G., \& Gotlib, I. (2014). Increased insula coactivation with salience networks in insomnia. Biological Psychology, 97, 1-8. http://dx.doi.org/10.1016/j.biopsycho.2013.12.016.

Daniels, M., McFarlane, A., Bluhm, R., Moores, K., Shaw, M., Williamson, P. et al. (2010). Switching between executive and default mode networks in posttraumatic stress disorder: alterations in functional connectivity. Journal of Psychiatry and Neuroscience, 35(4), 258-266.

De Havas, J., Parimal, S., Soon, C., \& Chee, M. (2012). Sleep deprivation reduces default mode network connectivity and anti-correlation during rest and task performance. NeuroImage, 59(2), 1745-1751. http://dx.doi.org/10.1016/j.neuroimage.2011.08.026.

Drake, C., \& Roth, T. (2006). Predisposition in the evaluation of insomnia: evidence, potential mechanisms and future directions. Sleep Medicine Clinics, 1, 333-349. http://dx.doi.org/10.1016/j.jsmc.2006.06.005.

Drummond, S., Walker, M., Almklov, E., Campos, M., Anderson, D., \& Straus, L. (2013). Neural correlates of working memory performance in primary insomnia. Sleep, 36, 1307-1316. http://dx.doi.org/10.5665/ sleep. 2952.

Espie, C., Broomfield, N., MacMahon, K., Macphee, L., \& Taylor, L. (2006). The attention-intention-effort pathway in the development of psychophysiologic insomnia: a theoretical review. Sleep Medicine Reviews, 10(21), 5-45. http://dx.doi.org/10.1016/j.smrv.2006.03.002.

Greicius, M., Flores, B., Menon, V., Glover, G., Solvason, H., \& Kenna, H. (2007). Resting-state functional connectivity in major depression: abnormally increased contributions from subgenual cingulate cortex and thalamus. Biological Psychiatry, 62(5), 429-437. http://dx.doi.org 10.1016/j.biopsych.2006.09.020.

Gujar, N., Yoo, S., Hu, P., \& Walker, M. (2010). The un-rested resting brain: sleep-deprivation alters activity within the default-mode network. Journal of Cognitive Neuroscience, 22(8), 1637-1648. http://dx.doi.org/ 10.1162/jocn.2009.21331.

Harvey, A. (2002). A cognitive model of insomnia. Behavior Research and Therapy, 40, 869-894. http://dx.doi.org/10.1016/S0005-7967(01) $00061-4$.

Harvey, A. (2005). Cognitive approaches to insomnia. Clinical Psychology Review, 25, 593-611. http://dx.doi.org/10.1016/j.cpr.2005.04.005.

Hasler, B., James, J., Franzen, P., Nofzinger, E., Germain, A., \& Buysse, D. (2013). Variation in default mode network connectivity across sleepwake states differs between adults with primary insomnia and good sleepers. Sleep, 36(abstract suppl.), 192.

Horovitz, S., Braun, A., Carr, W., Picchioni, D., Balkin, T., Fukunaga, M., et al. (2009). Decoupling of the brain's default mode network during deep sleep. Proceedings of the National Academy of Sciences of the United States of America, 106(27), 11376-11381. http://dx.doi.org/10.1073/ pnas.0901435106.

Kales, A., Caldwell, A., Preston, T., Healey, S., \& Kales, J. (1976). Personality patterns in insomnia: theoretical implications. Archives of General Psychiatry, 33, 1128-1134. http://dx.doi.org/10.1001/ archpsyc.1976.01770090118013.

Killingsworth, M., \& Gilbert, D. (2010). A wandering mind is an unhappy mind. Science, 330, 932. http://dx.doi.org/10.1126/science.1192439.

Knyazev, G., Slobodskoj-Plusnin, J., Bocharov, A., \& Pylkova, L. (2011). The default mode network and EEG alpha oscillations: an independent component analysis. Brain Research, 1402, 67-79. http://dx.doi.org/ 10.1016/j.brainres.2011.05.052.

Kornelsen, J., Sboto-Frankenstein, U., Mclver, Y., Gervai, P., Wacnik, P., Berrington, N., et al. (2013). Default mode network functional connectivity altered in failed back surgery syndrome. The Journal of Pain, 14(5), 483-491. http://dx.doi.org/10.1016/j.jpain.2012.12.018.

Larson-Prior, L., Zempel, J., Nolan, T., Prior, F., Snyder, A., \& Raichle, M. (2009). Cortical network functional connectivity in the descent to sleep. Proceedings of the National Academy of Sciences of the United States of America, 106(11), 4489-4494. http://dx.doi.org/10.1073/ pnas.0900924106.

Larson-Prior, L., Zempel, J., Prior, F., Vincent, J., Snyder, A., \& Raichle, M. (2006). Does alpha-band EEG activity in eyes-closed rest index a unitary state over time? NeuroImage, 31(Suppl. 1) http://dx.doi.org/ 10.1016/S1053-8119(08)70002-8, 187W-AM.

Lichstein, L., \& Rosenthal, T. (1980). Insomniacs' perceptions of cognitive versus somatic determinants of sleep disturbance. Journal of Abnormal Psychology, 89(1), 105-107. http://dx.doi.org/10.1037/0021843X.89.1.105.

Linden, D. (2006). How psychotherapy changes the brain: the contribution of functional neuroimaging. Molecular Psychiatry, 11, 528-538. http://dx.doi.org/10.1038/sj.mp.4001816.

Liu, P., Zeng, F., Zhou, G., Wang, J., Wen, H., von Deneen, K., et al. (2013). Alterations of the default mode network in functional dyspepsia patients: a resting-state fmri study. Neurogastroenterology \& Motility, 25(6), 382-388. http://dx.doi.org/10.1111/nmo.12131.

Lundh, L. (2005). The role of acceptance and mindfulness in the treatment of insomnia. Journal of Cognitive Psychotherapy: An International Quarterly, 19(1), 29-39. http://dx.doi.org/10.1891/jcop.19.1.29.66331.

Lundh, L., \& Broman, J. (2000). Insomnia as an interaction between sleepinterfering and sleep-interpreting processes. Journal of Psychosomatic Research, 49, 299-310. http://dx.doi.org/10.1016/S0022-3999(00) 00150-1.

Marchetti, I., Koster, E., Sonuga-Barke, E., \& Raedt, R. (2012). The default mode network and recurrent depression: a neurobiological model of cognitive risk factors. Neuropsychology Review, 22(3), 229-251. http:// dx.doi.org/10.1007/s11065-012-9199-9.

Morgenthaler, T., Kramer, M., Alessi, C., Friedman, L., Boehlecke, B. Brown, T., \&, AASM. (2006). Practice parameters for the psychological and behavioral treatment of insomnia: an update. Sleep, 29(11), 1415-1419.

Morin, C. (1993). Insomnia: Psychological assessment and management. New York, London: The Guilford Press.

Morin, C., Harvey, A., \& Bélanger, L. (2011). Comparative efficacy of behaviour therapy and cognitive therapy as single therapies for insomnia: a preliminary report. Sleep Medicine, 12(Suppl. 1), S5-S6.

Nofzinger, E. (2010). Brain imaging in insomnia. In M. Sateia, \& D. Buysse (Eds.), Insomnia: Diagnosis and treatment (pp. 77-83). UK: Informa Health Care.

Ong, J., Ulmer, C., \& Manber, R. (2012). Improving sleep with mindfulness and acceptance: a metacognitive model of insomnia. Behaviour 
Research and Therapy, 50, 651-660. http://dx.doi.org/10.1016/ j.brat.2012.08.001.

Paulesu, E., Sambugaro, E., Torti, T., Danelli, L., Ferri, F., Scialfa, G., \& Sassaroli, S. (2009). Neural correlates of worry in generalized anxiety disorder and in normal controls: a functional MRI study. Psychological Medicine, 40(1), 117-124. http://dx.doi.org/10.1017/S0033291709005649.

Perlis, M., Giles, D., Mendelson, W., Bootzin, R., \& Wyatt, J. (1997). Psychophysiological insomnia: the behavioural model and a neurocognitive perspective. Journal of Sleep Research, 6, 179-188. http:// dx.doi.org/10.1046/j.1365-2869.1997.00045.x.

Perlis, M., Shaw, P. Cano, G. \& Espie, C. (2011). Models of insomnia. In M. Kryger, T. Roth, \& W. Dement (Eds.), Principles and practice of sleep medicine (5th ed.). (pp. 850-865). Missouri: Elsevier Saunders.

Raichle, M., \& Snyder, A. (2007). A default mode of brain function: a brief history of an evolving idea. Neurolmage, 37, 1083-1090. http:// dx.doi.org/10.1016/j.neuroimage.2007.02.041.

Rao, H., Fang, Z., Hu, S., Zhu, S., Detre, J., Goel, N., et al. (2012, october). Reduced pcc-hippocampus connectivity predicts memory deficits after sleep deprivation. Paper presented at the annual meeting of the Society for Neuroscience, New Orleans.

Riemann, D., Spiegelhalder, K., Feige, B., Voderholzer, U., Berger, M., Perlis, M., et al. (2010). The hyperarousal model of insomnia: a review of the concept and its evidence. Sleep Medicine Reviews, 14, 19-31. http://dx.doi.org/10.1016/j.smrv.2009.04.002.

Riemann, D., Spiegelhalder, K., Nissen, C., Hirscher, V., Baglioni, C., \& Feige, B. (2012). REM sleep instability: a new pathway for insomnia? Pharmacopsychiatry, 45(5), 167-176. http://dx.doi.org/10.1055/s0031-1299721.

Sämann, P., Tully, C., Spoormaker, V., Wetter, T., Holsboer, F., Wehrle, R., et al. (2010). Increased sleep pressure reduces resting state functional connectivity. Magnetic Resonance Materials in Physics, Biology and Medicine, 23, 375-389. http://dx.doi.org/10.1007/s10334-010-0213-z.

Sämann, P., Wehrle, R., Hoehn, D., Spoormaker, V., Peters, H., Tully, C., et al. (2011). Development of the brain's default mode network from wakefulness to slow wave sleep. Cerebral Cortex. http://dx.doi.org/ 10.1093/cercor/bhq295.

Sarsam, M., Parkes, L., Roberts, N., Reid, G., \& Kinderman, P. (2013). The queen and I: neural correlates of altered self-related cognitions in major depressive episode. Plos One, 8(10), e78844. http://dx.doi.org/ 10.1371/journal.pone.0078844.

Schacter, D., Addis, D., \& Buckner, R. (2007). Remembering the past to imagine the future: the prospective brain. Nature Reviews, 8, 657-661. http://dx.doi.org/10.1038/nrn2213.
Snyder, A., \& Raichle, M. (2012). A brief history of the resting state: the washington university perspective. NeuroImage, 62, 902-910. http:// dx.doi.org/10.1016/j.neuroimage.2012.01.044.

Spielman, A., Caruso, L., \& Glovinsky, P. (1987). A behavioral perspective on insomnia treatment. Psychiatric Clinical North American, 10, 541-553.

Tagliazucchi, E., \& Laufs, H. (2014). Decoding wakefulness levels from typical fMRI resting-state data reveals reliable drifts between wakefulness and sleep. Neuron, 82(3), 695-708. http://dx.doi.org/10.1016/ j.neuron.2014.03.020.

Talbot, L., \& Harvey, A. (2010). Psychological models of insomnia. In M. Sateia, \& D. Buysse (Eds.), Insomnia: Diagnosis and treatment (pp. 42-49). UK: Informa Health Care.

Tregellas, J., Wylie, K., Rojas, D., Tanable, J., Martin, J., Kronberg, E., et al. (2011). Altered default network activity in obesity. Integrative Physiology, 19(12), 2316-2321. http://dx.doi.org/10.1038/oby.2011.119.

Violante, I., Ribeiro, M., Cunha, G., Bernardino, I., Duarte, J., Ramos, F., et al. (2012). Abnormal brain activation in neurofibromatosis type 1: a link between visual processing and the default mode network. PLoS One, 7(6). http://dx.doi.org/10.1371/journal.pone.0038785.

Wamsley, E., \& Stickgold, R. (2011). Memory, sleep and dreaming: experiencing consolidation. Sleep Medicine Clinics, 6(1), 97-108.

Watts, F., Coyle, K., \& East, M. (1994). The contribution of worry to insomnia. British Journal of Clinical Psychology, 33, 211-220. http:// dx.doi.org/10.1111/j.2044-8260.1994.tb01115.x.

Whitfield-Gabrieli, S., \& Ford, J. (2012). Default mode network activity and connectivity in psychopathology. Annual Review of Clinical Psychology, 8, 49-76. http://dx.doi.org/10.1146/annurev-clinpsy-032511-143049.

Wolf, R., Sambataro, F., Vasic, N., Wolf, N., Thomann, P., Saft, C., et al. (2012). Default-mode network changes in preclinical Huntington's disease. Experimental Neurology, 237(1), 191-198. http://dx.doi.org/ 10.1016/j.expneurol.2012.06.014.

Xu, P., Huang, R., Wang, J., Van Dam, N., Xie, T., Dong, Z., et al. (2014). Different topological organization of human brain functional networks with eyes open versus eyes closed. NeuroImage, 90, 246-255. http://dx.doi.org/10.1016/j.neuroimage.2013.12.060.

Zhao, X., Wang, P., Li, C., Hu, Z., Xi, Q., Wu, W., et al. (2007). Altered default mode network activity in patient with anxiety disorders: an fMRI study. European Journal of Radiology, 63(3), 373-378. http:// dx.doi.org/10.1016/j.ejrad.2007.02.006. 